\title{
eToxPred: a machine learning-based approach to estimate the toxicity of drug candidates
}

\author{
Limeng Pu', Misagh Naderi' ${ }^{2}$ Tairan $\mathrm{Liu}^{3}$, Hsiao-Chun $\mathrm{Wu}^{1}$, Supratik Mukhopadhyay ${ }^{4}$ and Michal Brylinski ${ }^{2,5^{*}}$ (D)
}

\begin{abstract}
Background: The efficiency of drug development defined as a number of successfully launched new pharmaceuticals normalized by financial investments has significantly declined. Nonetheless, recent advances in high-throughput experimental techniques and computational modeling promise reductions in the costs and development times required to bring new drugs to market. The prediction of toxicity of drug candidates is one of the important components of modern drug discovery.

Results: In this work, we describe eToxPred, a new approach to reliably estimate the toxicity and synthetic accessibility of small organic compounds. eToxPred employs machine learning algorithms trained on molecular fingerprints to evaluate drug candidates. The performance is assessed against multiple datasets containing known drugs, potentially hazardous chemicals, natural products, and synthetic bioactive compounds. Encouragingly, eToxPred predicts the synthetic accessibility with the mean square error of only $4 \%$ and the toxicity with the accuracy of as high as $72 \%$.

Conclusions: eToxPred can be incorporated into protocols to construct custom libraries for virtual screening in order to filter out those drug candidates that are potentially toxic or would be difficult to synthesize. It is freely available as a stand-alone software at https://github.com/pulimeng/etoxpred.
\end{abstract}

Keywords: Virtual screening, Synthetic accessibility, Toxicity, Machine learning, Deep belief network, Extremely randomized trees

\section{Background}

Drug discovery is an immensely expensive and time-consuming process posing a number of formidable challenges. To develop a new drug requires $6-12$ years and costs as much as $\$ 2.6$ billion [1, 2]. These expenses do not include the costs of basic research at the universities focused on the identification of molecular targets, and the development of research methods and technologies. Despite this cumbersome discovery process, the pharmaceutical industry is still regarded as highly profitable because the expenses are eventually accounted for in the market price of new therapeutics. Although, a breakdown

\footnotetext{
*Correspondence: michal@brylinski.org

2Department of Biological Sciences, Louisiana State University, Baton Rouge, LA 70803, USA

${ }^{5}$ Center for Computation \& Technology, Louisiana State University, Baton

Rouge, LA 70803, USA

Full list of author information is available at the end of the article
}

of the overall capitalized costs shows that the clinical period costing $\$ 1.5$ billion is economically the most critical factor, the expenditures of the pre-human phase aggregate to $\$ 1.1$ billion [1]. Thus, technological advances in discovery research and preclinical development could potentially lower the costs of bringing a new drug to the market.

Computer-aided drug discovery (CADD) holds a significant promise to reduce the costs and speed up the development of lead candidates at the outset of drug discovery [3]. Powered by continuous advances in computer technologies, CADD employing virtual screening (VS) allows identifying hit compounds from large databases of drug-like molecules much faster than traditional approaches. CADD strategies include ligand- and structure-based drug design, lead optimization, and the comprehensive evaluation of absorption, distribution, metabolism, excretion, and toxicity (ADMET) parameters [4]. Ligand-based drug design 
(LBDD) leverages the spatial information and physicochemical features extracted from known bioactives against a given target protein to design and optimize new compounds for the same target [5]. VS employing features provided by pharmacophore modeling [6] and quantitative structure-activity relationship (QSAR) analysis [7] can be performed in order to identify potentially active compounds. Although the capabilities of the traditional LBDD to discover new classes of leads may be limited, recent advances in generating targeted virtual chemical libraries by combinatorial chemistry methods considerably extend the application of LBDD methods [8-10]. Captopril, an angiotensin-converting enzyme inhibitor, was one of the first success stories of LBDD, which was considered a revolutionary concept in 1970s compared to conventional methods [11].

Although the combination of pharmacophore modeling, QSAR, and VS techniques has been demonstrated to be valuable in the absence of the protein structure data [12, 13], the three-dimensional (3D) information on the target protein allows employing structure-based drug design (SBDD) [14] in CADD. Foremost SBDD methods include molecular docking [15], molecular dynamics [16], receptor-based VS [17], and the de novo design of active compounds [18]. Molecular docking is widely used in CADD to predict the preferable orientation of a drug molecule in the target binding pocket by finding the lowest energy configuration of the protein-ligand system. It is often employed to conduct receptor-based VS whose goal is to identify in a large library of candidate molecules those compounds that best fit the target binding site. VS performed with high-performance computing machines renders docking programs such as AutoDock Vina [19], rDock [20], Glide [21], and FlexX [22] capable to search through millions of compounds in a matter of days or even hours. A potent, pyrazole-based inhibitor of the transforming growth factor- $\beta$ type I receptor kinase exemplifies benefits of utilizing receptor-based VS to discover leads. This inhibitor has been independently discovered with the computational, shape-based screening of 200,000 compounds [23] as well as the traditional enzyme and cell-based high-throughput screening of a large library of molecules [24].

In addition to LBDD and SBDD, toxicity prediction is an increasingly important component of modern CADD, especially considering that the collections of virtual molecules for VS may comprise tens of millions of untested compounds. Methods to predict toxicity aim at identifying undesirable or adverse effects of certain chemicals on humans, animals, plants, or the environment. Conventional approaches to evaluate toxicity profiles employing animal tests are constrained by time, costs, and ethical considerations. On that account, fast and inexpensive computational approaches are often employed at first in order to eliminate potentially toxic molecules and reduce the number of experimental tests that need to be conducted. For instance, a blockage of the human Ether-à-go-go-Related Gene (hERG) potassium ion channels by a surprisingly diverse group of drugs can induce lethal cardiac arrhythmia [25]. Therefore, the effective identification of putative hERG blockers and non-blockers in chemical libraries plays an important role in the cardiotoxicity prediction. A recently developed method, Pred-hERG, estimates the cardiac toxicity with a set of features based on statistically significant and externally predictive QSAR models of the hERG blockage [26]. Pred-hERG employs a binary model, a multi-class model, and the probability maps of atomic contribution, which are combined for the final prediction. Encouragingly, Pred-hERG achieves a high correct classification rate of 0.8 and a multi-class accuracy of 0.7 .

Another example is chemTox (http://www.cyprotex.com/ insilico/physiological_modelling/chemtox) predicting key toxicity parameters, the Ames mutagenicity [27] and the median lethal dose $\left(\mathrm{LD}_{50}\right)$ following intravenous and oral administration, as well as the aqueous solubility. chemTox employs molecular descriptors generated directly from chemical structures to construct quantitative-structure property relationships (QSPR) models. Since this method requires a set of specific descriptors to generate QSPR models for a particular type of toxicity, it may not be suitable to evaluate a broadly defined toxicity and drug side-effects in general. A similar method, ProTox, predicts rodent oral toxicity based on the analysis of toxic fragments present in compounds with known $\mathrm{LD}_{50}$ values [28]. ProTox additionally evaluates possible targets associated with adverse drug reactions and the underlying toxicity mechanisms with the collection of protein-ligand pharmacophores, called toxicophores. This tool was reported to outperform the commercial software TOPKAT (TOxicity Prediction by Komputer Assisted Technology, http://accelrys.com/products/collaborative-science/biovia-discovery-studio/qsar-admet-and-predictive-toxicology.html) against a diverse external validation set, with the sensitivity, specificity and precision of $0.76,0.95$ and 0.75 , respectively. Other techniques to predict toxicity utilize various features such as fingerprints, physicochemical properties, and pharmacophore models to build predictive dose- and time-response models [29].

The Tox21 Data Challenge 2014 (https://tripod.nih.gov/ tox21/challenge/index.jsp) has been conducted to assess a number of methods predicting how chemical compounds disrupt biological pathways in ways that may result in toxic effects. In this challenge, the chemical structure data for 12,707 compounds were provided in order to evaluate the capabilities of modern computational approaches to identify those environmental chemicals and drugs that are of the greatest potential concern to human health. DeepTox 
[30] was the best performing methods in the Tox21 Data Challenge winning the grand challenge, the nuclear receptor panel, the stress response panel, and six single assays. This algorithm employs the normalized chemical representations of compounds to compute a large number of descriptors as an input to machine learning. Models in DeepTox are first trained and evaluated, and then the most accurate models are combined into ensembles ultimately used to predict the toxicity of new compounds. DeepTox was reported to outperform deep neural networks (DNNs) [31], support vector machines (SVMs) [32], random forests (RF) [33], and elastic nets [34].

In this communication, we describe eToxPred, a new method to predict the synthetic accessibility and the toxicity of molecules in a more general manner. In contrast to other approaches employing manually-crafted descriptors, e ToxPred implements a generic model to estimate the toxicity directly from the molecular fingerprints of chemical compounds. Consequently, it may be more effective against highly diverse and heterogeneous datasets. Machine learning models in $e$ ToxPred are trained and cross-validated against a number of datasets comprising known drugs, potentially hazardous chemicals, natural products, and synthetic bioactive compounds. We also conduct a comprehensive analysis of the chemical composition of toxic and non-toxic substances. Overall, $e$ ToxPred quite effectively estimates the synthetic accessibility and the toxicity of small organic compounds directly from their molecular fingerprints. As the primary application, this technique can be incorporated into high-throughput pipelines constructing custom libraries for virtual screening, such as that based on eMolFrag [9] and $e$ Synth [10], to eliminate from CADD those drug candidates that are potentially toxic or would be difficult to synthesize.

\section{Implementation}

\section{Machine learning algorithms}

Numerous machine learning-based techniques have been developed to reveal complex relations between chemical entities and their biological targets [35]. In Fig. 1, we briefly present the concepts and the overall implementation of machine learning classifiers employed in this study. The first algorithm is the Restricted Boltzmann Machine (RBM), an undirected graphical model with a visible input layer and a hidden layer. In contrast to the unrestricted Boltzmann Machine, in which all nodes are connected to one another (Fig. 1A) [36], all inter-layer units in the RBM are fully connected, while there are no intra-layer connections (Fig. 1B) [37]. The RBM is an energy-based model capturing dependencies between variables by assigning an "energy" value to each configuration. The RBM is trained by balancing the probability of various regions of the state space, viz. the energy of those regions with a high probability is reduced, with the simultaneous increase in the energy of low-probability regions. The training process involves the optimization of the weight vector through Gibbs sampling [38].
A

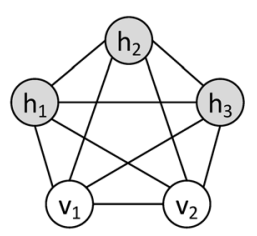

B

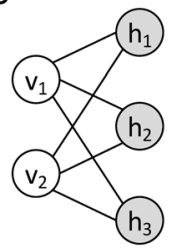

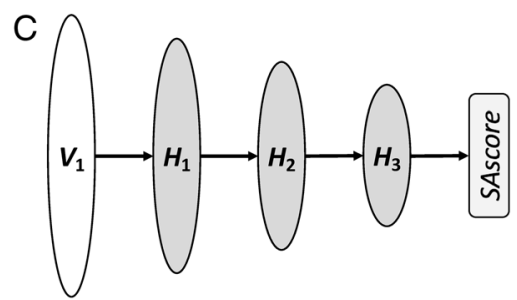

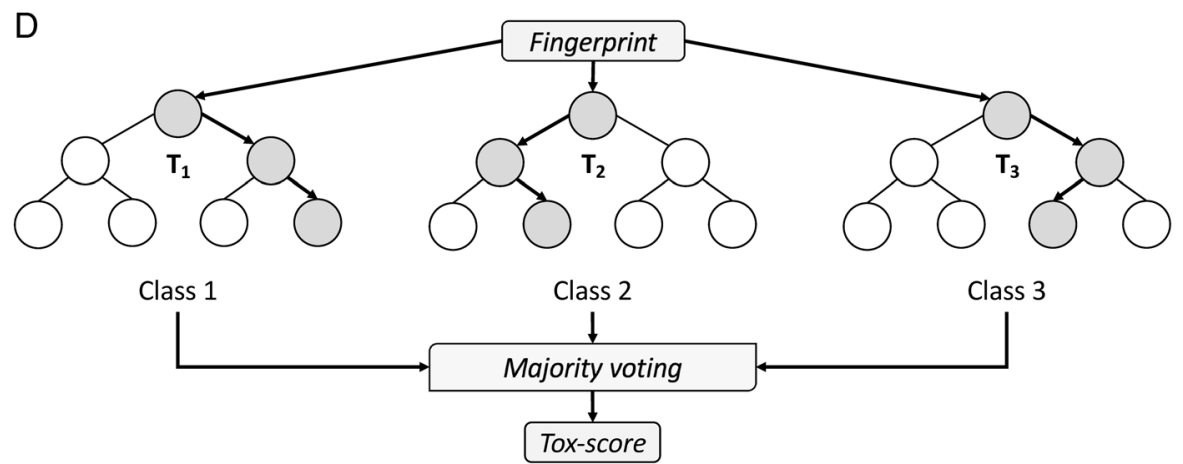

Fig. 1 Schematics of various machine learning classifiers. (a) A two-layered Boltzmann Machine with 3 hidden nodes $h$ and 2 visible nodes $v$. Nodes are fully connected. (b) A Restricted Boltzmann Machine (RBM) with the same nodes as in A. Nodes belonging to the same layer are not connected. (c) A Deep Belief Network with a visible layer $V$ and 3 hidden layers $H$. Individual layers correspond to RBMs that are stacked against one another. (d) A Random Forest with 3 trees T. For a given instance, each tree predicts a class based on a subset of the input set. The final class assignment is obtained by the majority voting of individual trees 
The Deep Belief Network (DBN) is a generative probabilistic model built on multiple RBM units stacked against each other, where the hidden layer of an unsupervised RBM serves as the visible layer for the next sub-network (Fig. 1C) [39]. This architecture allows for a fast, layer-by-layer training, during which the contrastive divergence algorithm [40] is employed to learn a layer of features from the visible units starting from the lowest visible layer. Subsequently, the activations of previously trained features are treated as a visible unit to learn the abstractions of features in the successive hidden layer. The whole DBN is trained when the learning procedure for the final hidden layer is completed. It is noteworthy that DBNs are first effective deep learning algorithms capable of extracting a deep hierarchical representation of the training data [41].

In this study, we utilize a DBN implemented in Python with Theano and CUDA to support Graphics Processing Units (GPUs) [42]. The SAscore is predicted with a DBN architecture consisting of a visible layer corresponding to a 1024-bit Daylight fingerprint (http://www.daylight.com) and three hidden layers having 512, 128, and 32 nodes (Fig. 1C). The L2 regularization is employed to reduce the risk of overfitting. The DBN employs an adaptive learning rate decay with an initial learning rate, a decay rate, mini-batch size, the number of pre-training epochs, and the number of fine-tuning epochs of 0.01 , $0.0001,100,20$, and 1000, respectively.

Finally, the Extremely Randomized Trees, or Extra Trees (ET), algorithm [43] is used to predict the toxicity of drug candidates (Fig. 1D). Here, we employ a simpler algorithm because classification is generally less complex than regression. Classical random decision forests construct an ensemble of unpruned decision trees predicting the value of a target variable based on several input variables [44]. Briefly, a tree is trained by recursively partitioning the source set into subsets based on an attribute value test. The dataset fits well the decision tree model because each feature takes a binary value. The recursion is completed when either the subset at a node has an invariant target value or when the Gini impurity reaches a certain threshold [45]. The output class from a decision forest is simply the mode of the classes of the individual trees. The ET classifier is constructed by adding a randomized top-down splitting procedure in the tree learner. In contrast to other tree-based methods commonly employing a bootstrap replica technique, ET splits nodes by randomly choosing both attributes and cut-points, as well as it uses the whole learning sample to grow the trees. Random decision forests, including ET, are generally devoid of problems caused by overfitting to the training set because the ensemble of trees reduces model complexity leading to a classifier with a low variance. In addition, with a proper parameter tuning, the randomization procedure in ET can help achieve robust performance even for small training datasets.

The ET classifier used in this study is implemented in Python. We found empirically that the optimal performance in terms of the out-of-bag error is reached at 500 trees and adding more trees causes overfitting and increases the computational complexity. The number of features to be randomly drawn from the 1024-bit input vector is $\log _{2} 1024=10$. The maximum depth of a tree is 70 with minimum numbers of 3 and 19 samples to create and split a leaf node, respectively.

\section{Datasets}

Table 1 presents compound datasets are employed in this study. The first two sets, the Nuclei of Bioassays, Ecophysiology and Biosynthesis of Natural Products (NuBBE), and the Universal Natural Products Database (UNPD), are collections of natural products. NuBBE is a virtual database of natural products and derivatives from the Brazilian biodiversity [46], whereas UNPD is a general resource of natural products created primarily for virtual screening and network pharmacology [47]. Removing the redundancy at a Tanimoto coefficient (TC) [48] of 0.8 with the SUBSET [49] program resulted in 1008 NuBBE and 81,372 UNPD molecules. In addition to natural products, we compiled a non-redundant set of mostly synthetic bioactive compounds from the Database of Useful Decoys, Extended (DUD-E) database [50] by selecting 17,499 active molecules against 101 pharmacologically relevant targets.

The next two sets, FDA-approved and Kyoto Encyclopedia of Genes and Genomes (KEGG) Drug, comprise molecules approved by regulatory agencies, which possess acceptable risk versus benefit ratios. Although these molecules may still cause adverse drug reactions, we refer to them as non-toxic because of their relatively high therapeutic indices. FDA-approved drugs were obtained from the DrugBank database, a widely used cheminformatics resource providing comprehensive information on known drugs and their molecular targets [51]. The KEGG-Drug resource contains drugs approved in Japan, United States, and Europe, annotated with the information on their targets, metabolizing enzymes, and molecular interactions [52]. Removing the chemical redundancy from both datasets yielded 1515 FDA-approved and 3682 KEGG-Drug compounds.

Two counter-datasets, TOXNET and the Toxin and Toxin Target Database (T3DB), contain compounds indicated to be toxic. The former resource maintained by the National Library of Medicine provides databases on toxicology, hazardous chemicals, environmental health, and toxic releases [53]. Here, we use the Hazardous Substances Data Bank focusing on the toxicology of potentially hazardous chemicals. T3DB houses detailed toxicity 
Table 1 Compound datasets used to evaluate the performance of eToxPred. These non-redundant sets are employed to train and test SAscore, Tox-score, and specific toxicities

\begin{tabular}{llll}
\hline Dataset & Size & Usage & Description \\
\hline NuBBE & 1008 & Train/test (SAscore) & Natural products and derivatives from the Brazilian biodiversity \\
UNPD & 81,372 & Train/test (SAscore) & Diverse collection of natural products \\
DUD-E (actives) & 17,499 & Train/test (SAscore) & Mostly synthetic bioactive compounds against 102 protein targets \\
FDA-approved & 1515 & $\begin{array}{l}\text { Train/test (SAscore) } \\
\text { Train (Tox-score) }\end{array}$ & FDA approved drugs from DrugBank \\
KEGG-Drug & 3682 & Test (Tox-score) & Drugs approved in Japan, United States, and Europe \\
TOXNET & 3035 & Train (Tox-score) & Potentially hazardous chemicals \\
T3DB & 1283 & Test (Tox-score) & Collection of pollutants, pesticides, drugs, and food toxins \\
TCM & 5883 & Test (SAscore, Tox-score, unlabeled) & Traditional Chinese medicines \\
CP & 1401 & Train/test (specific toxicity) & Carcinogenic compounds tested in rodents \\
CD & 1571 & Train/test (specific toxicity) & Cardiotoxic compounds tested against hERG potassium channel \\
ED & 17,059 & Train/test (specific toxicity) & Endocrine disrupting compounds tested against androgen and \\
AO & 12,612 & Train/test (specific toxicity) & estrogen receptors
\end{tabular}

data in terms of chemical properties, molecular and cellular interactions, and medical information, for a number of pollutants, pesticides, drugs, and food toxins [54]. These data are extracted from multiple sources including other databases, government documents, books, and scientific literature. The non-redundant sets of TOXNET and T3DB contain 3035 and 1283 toxic compounds, respectively.

As an independent set, we employ the Traditional Chinese Medicine (TCM) Database@Taiwan, currently the largest and most comprehensive small molecule database on traditional Chinese medicine for virtual screening [55]. TCM is based on information collected from Chinese medical texts and scientific publications for 453 different herbs, animal products, and minerals. From the original dataset, we first selected molecules with a molecular weight in the range of $100-600 \mathrm{Da}$, and then removed redundancy at a TC of 0.8 , producing a set of 5883 unique TCM compounds.

Finally, we use four datasets to evaluate the prediction of specific toxicities. Compounds causing cancer in high dose tests were obtained from the Carcinogenicity Potency (CP) database [56]. These data are labeled based on series of experiments carried out on rodents considering different tissues of the subjects. A chemical is deemed toxic if it caused tumor growth in at least one tissue specific experiment. The CP set comprises 796 toxic and 605 non-toxic compounds. The cardiotoxicity (CD) dataset contains 1571 molecules characterized with bioassay against human ether-a-go-go related gene (hERG) potassium channel. hERG channel blockade induces lethal arrhythmia causing a life-threatening symptom [57]. The CD set includes 350 toxic compounds with an $\mathrm{IC}_{50}$ of $<1 \mu \mathrm{M}$ [58]. The endocrine disruption (ED) dataset is prepared based on the bioassay data for androgen and estrogen receptors taken from the Tox21 Data Challenge. Endocrine disrupting chemicals interfere with the normal functions of endogenous hormones causing metabolic and reproductive disorders, the dysfunction of neuronal and immune systems, and cancer growth [59]. The ED set contains 1317 toxic and 15,742 non-toxic compounds. The last specific dataset is focused on the acute oral toxicity (AO). Among 12,612 molecules with $\mathrm{LD}_{50}$ data provided by the SuperToxic database [60], 7392 compounds are labeled as toxic with a $\mathrm{LD}_{50}$ of $<500$ $\mathrm{mg} \mathrm{kg}{ }^{-1}$. It is important to note that since $L D_{50}$ is not indicative of non-lethal toxic effects, a chemical with a high $\mathrm{LD}_{50}$ may still cause adverse reactions at small doses.

\section{Model training, cross-validation, and evaluation}

Input data to machine learning models are 1024-bit Daylight fingerprints constructed for dataset compounds with Open Babel [61]. The reference SAscore values are computed with an exact approach that combines the fragment-based score representing the "historical synthetic knowledge" with the complexity-based score penalizing the presence of ring systems, such as spiro and fused rings, multiple stereo centers, and macrocycles [62]. The DBN-based predictor of the SAscore was trained and cross-validated against NuBBE, UNPD, FDA-approved, and DUD-E-active datasets. Cross-validation is a common technique used in statistical learning to evaluate the generalization of a trained model [63]. In a $k$-fold cross-validation protocol, one first divides the dataset into $k$ different subsets and then the first subset is used as a validation set for a model trained on the remaining $k-1$ subsets. This procedure is repeated $k$ times employing different subsets as the validation set. Averaging the performance obtained for all $k$ subsets yields the overall performance and estimates the validation error of the model. In this 
work, the SAscore predictor is evaluated with a 5-fold cross-validation protocol, which was empirically demonstrated to be sufficient for most applications [64].

The Tox-score prediction is conducted with a binary, ET-based classifier. The training and cross-validation are carried out for the FDA-approved dataset used as positive (non-toxic) instances and the TOXNET dataset used as negative (toxic) instances. Subsequently, the toxicity predictor is trained on the entire FDA-approved/TOXNET dataset and then independently tested against the KEGG-Drug (positive, non-toxic) and T3DB (negative, toxic) sets. In addition, the capability of the classifier to predict specific toxicities is assessed against $\mathrm{CP}, \mathrm{CD}, \mathrm{ED}$, and $\mathrm{AO}$ datasets. Similar to the SAscore predictor, a 5 -fold cross-validation protocol is employed to rigorously evaluate the performance of the toxicity classifier. Finally, both machine learning predictors of SAscore and Tox-score are applied to the TCM dataset.

The performance of $e$ ToxPred is assessed with several metrics derived from the confusion matrix, the accuracy (ACC), the sensitivity or true positive rate (TPR), and the fall-out or false positive rate (FPR):

$$
\begin{aligned}
& \mathrm{ACC}=\frac{T P+T N}{T P+F P+T N+F N} \\
& \mathrm{TPR}=\frac{T P}{T P+F N} \\
& \mathrm{FPR}=\frac{F P}{F P+T N}
\end{aligned}
$$

where $T P$ is the number of true positives. i.e. non-toxic compounds classified as non-toxic, and $T N$ is the number of true negatives, i.e. toxic compounds classified as toxic. FP and $F N$ are the numbers of over- and under-predicted non-toxic molecules, respectively.

In addition, we assess the overall quality of a binary classifier with the Matthews correlation coefficient (MCC) [65] and the Receiver Operating Characteristic (ROC) analysis. The MCC is generally regarded as a well-balanced measure ranging from - 1 (anti-correlation) to 1 (a perfect classifier) with values around 0 corresponding to a random guess:

$$
\mathrm{MCC}=\frac{T N \times T P-F P \times F N}{\sqrt{(T P+F P)(T P+F N)(T N+F P)(T N+F N)}}
$$

where $T P, T N, F P$, and $F N$ are defined above. The ROC analysis describes a trade-off between the FPR and the
TPR for a classifier at varying decision threshold values. The MCC and ROC are important metrics to help select the best model considering the cost and the class distribution. The hyperparameters of the model, including the number of features resulting in the best split, the minimum number of samples required to split an internal node, and the minimum number of samples required to be at a leaf node, are tuned with a grid search method. The best set of hyperparameters maximizes both the MCC and ROC.

Finally, the performance of the regression classifier is evaluated with the mean squared error (MSE) and the Pearson correlation coefficient (PCC) [66]. The MSE is a risk function measuring the average of the squares of the errors:

$$
\mathrm{MSE}=\frac{1}{N} \sum_{i=1}^{N}\left(\widehat{y}_{i}-y_{i}\right)^{2}
$$

where $N$ is the total number of evaluation instances, and $\widehat{y}_{i}$ and $y_{i}$ are the predicted and actual values of $i$-th instance, respectively. Further, the PCC is often employed to assess the accuracy of point estimators by measuring the linear correlation between the predicted and actual values. Similar to the MCC, PCC ranges from -1 to 1 , where -1 is a perfect anti-correlation, 1 is a perfect correlation, and 0 is the lack of any correlation. It is calculated as:

$$
\mathrm{PCC}=\frac{\operatorname{cov}(\hat{y}, y)}{\sigma_{\hat{y}} \sigma_{y}}
$$

where $\operatorname{cov}(\hat{y}, y)$ is the covariance matrix of the predicted and actual values, and $\sigma_{\hat{y}}$ and $\sigma_{y}$ are the standard deviations of the predicted and actual values, respectively.

\section{Results and discussion \\ SAscore prediction with eToxPred}

The SAscore combining contributions from various molecular fragments and a complexity penalty, was developed to help estimate the synthetic accessibility of organic compounds [62]. It ranges from 1 for molecules easy to make, up to 10 for those compounds that are very difficult to synthetize. The datasets used to train and validate the SAscore predictor, including FDA-approved, DUD-E-active, NuBBE, and UNPD datasets, are highly skewed, i.e., SAscore values are non-uniformly distributed over the 1-10 range. For instance, Fig. 2 (solid gray line) shows that as many as $28.3 \%$ of molecules in the original dataset have a SAscore between 2 and 3. Therefore, a pre-processing is needed to balance the dataset for a better performance of the SAscore predictor. Specifically, an 


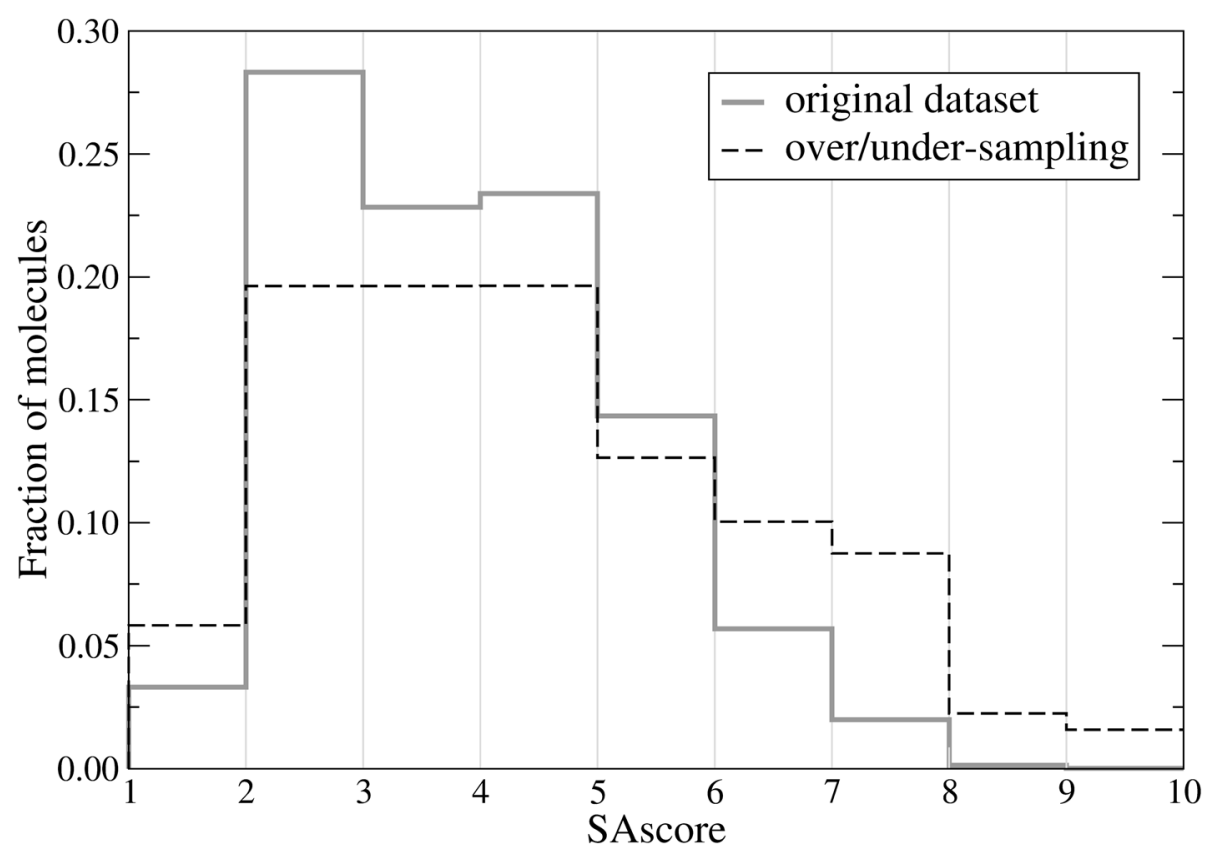

Fig. 2 Resampling strategy to balance the dataset. The histogram shows the distribution of SAscore values across the training set before (solid gray line) and after (dashed black line) the over/under-sampling

over/under-sampling procedure is employed by duplicating those cases with under-represented SAscore values and randomly selecting a subset of over-represented instances. The over-sample ratio for the $1-2$ range is 2 . The number of data points in the $2-5$ range are uniformly under-sampled to 90,000 , whereas those in the 5-6 range remain unchanged. For $6-7,7-8,8-9$, and 9-10 ranges, the over-sample ratios are $2,5,20$, and 100, respectively. Figure 2 (dashed black line) shows that the over/under-sampled set contains more instances with low (1-2) and high (6-10) SAscore values compared to the original dataset.

A scatter plot of the predicted vs. actual SAscore values is shown in Fig. 3 for FDA-approved, DUD-E-active, NuBBE, and UNPD datasets. Encouragingly, the cross-validated PCC (Eq. 6) across all four datasets is as high as 0.89 with a low MSE (Eq. 5) of 0.81 $(\sim 4 \%)$ for the predicted SAscore. Next, we apply the DBN predictor to individual datasets and analyze the distribution of the estimated SAscore values in Fig. 4. As expected, mostly synthetic molecules from the DUD-Eactive dataset have the lowest median SAscore of 2.9, which is in line with values previously reported for catalogue and bioactive molecules from the World Drug Index (http://www.daylight.com/products/wdi.html) and MDL Drug Data Report (http://www.akosgmbh.de/accelrys/databases/mddr.htm) databases. The median SAscore for FDA-approved drugs is 3.2 because in addition to synthetic and semi-synthetic compounds, this heterogeneous dataset also contains natural products whose chemical structures are generally more complex than the "standard" organic molecules. Both datasets of natural products, NuBBE and UNPD, have even higher median SAscore values of 3.4 and 4.1, respectively. Further, similar to the analysis of the Dictionary of Natural Products (http:// dnp.chemnetbase.com) conducted previously [62], natural products employed in the present study have a characteristic bimodal distribution with two distinct peaks at a SAscore of about 3 and 5. Finally, the median SAscore for TCM is 4.1 concurring with those values calculated for natural products. Interestingly, a number of TCM molecules have relatively high synthetic accessibility and the shape of the distribution of the estimated SAscore values is similar to that for the active compounds from the DUD-E dataset. Overall, the developed DBN-based model is demonstrated to be highly effective in estimating the SAscore directly from binary molecular fingerprints.

\section{Tox-score prediction with eToxPred}

$e$ ToxPred was developed to quickly estimate the toxicity of large collections of low molecular weight organic compounds. It employs an ET classifier to compute the Tox-score ranging from 0 (a low probability to be toxic) to 1 (a high probability to be toxic). The primary dataset to evaluate $e$ ToxPred consists of FDA-approved drugs, considered to be non-toxic, and potentially hazardous chemicals from the TOXNET database. Figure 5 shows the cross-validated performance of $e$ ToxPred in the prediction of toxic molecules. The ROC curve in Fig. 5A demonstrates that the ET classifier is highly accurate 


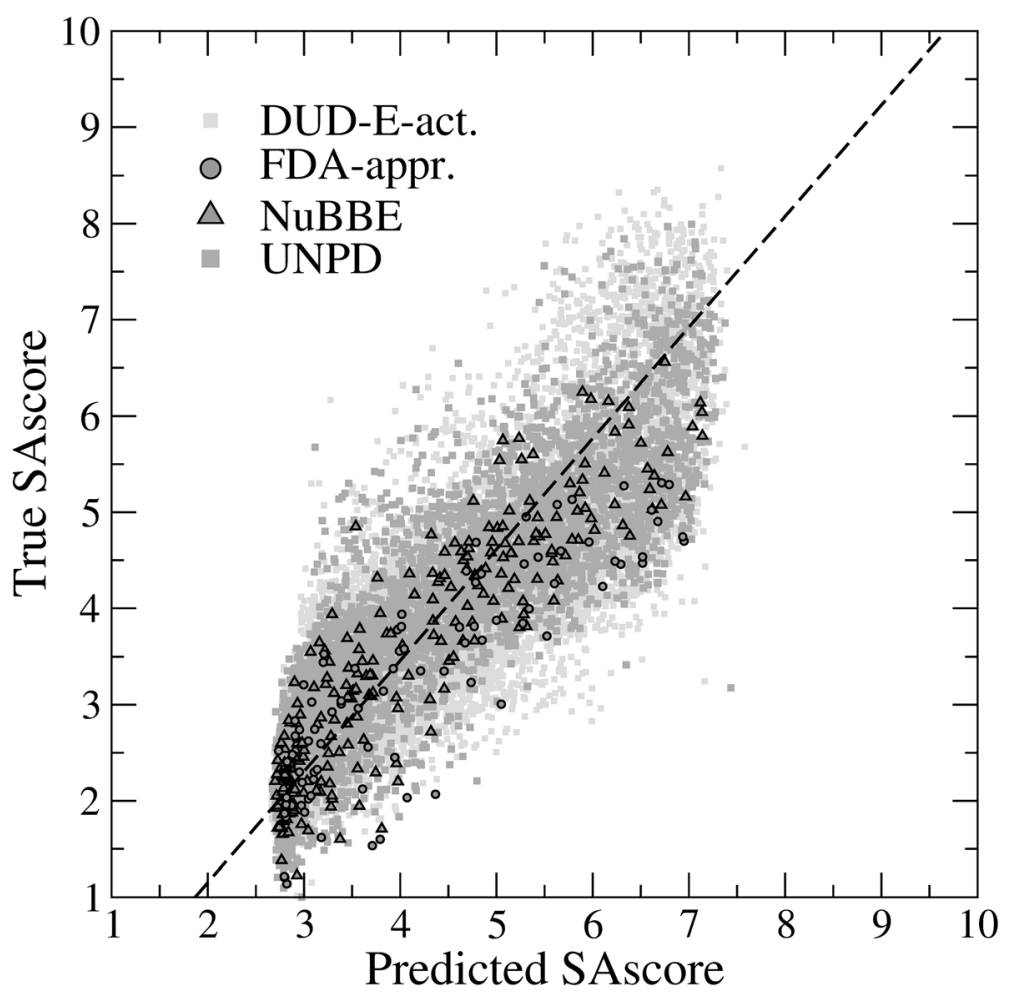

Fig. 3 SAscore prediction for several datasets. The scatter plot shows the correlation between the predicted and true SAscore values for active compounds from the Directory of Useful Decoys, Extended (DUD-E), FDA-approved drugs, and natural products from the NuBBE and UNPD databases. The regression line is dashed black
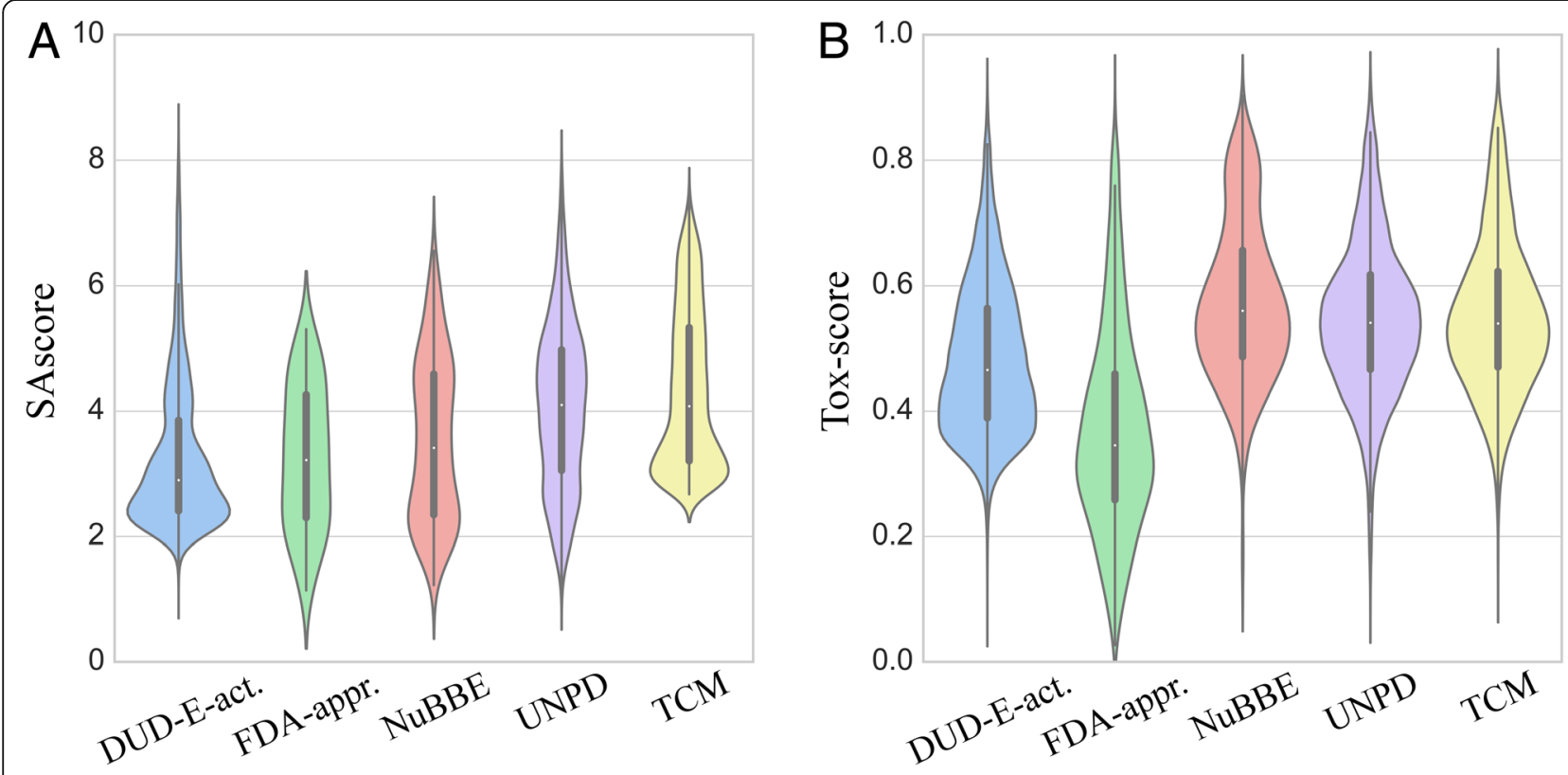

Fig. 4 SAscore and Tox-score prediction for several datasets. Violin plots show the distribution of (a) SAscore and (b) Tox-score values across active compounds from the Directory of Useful Decoys, Extended (DUD-E), FDA-approved drugs, natural products from the NuBBE and UNPD databases, and traditional Chinese medicines (TCM) 


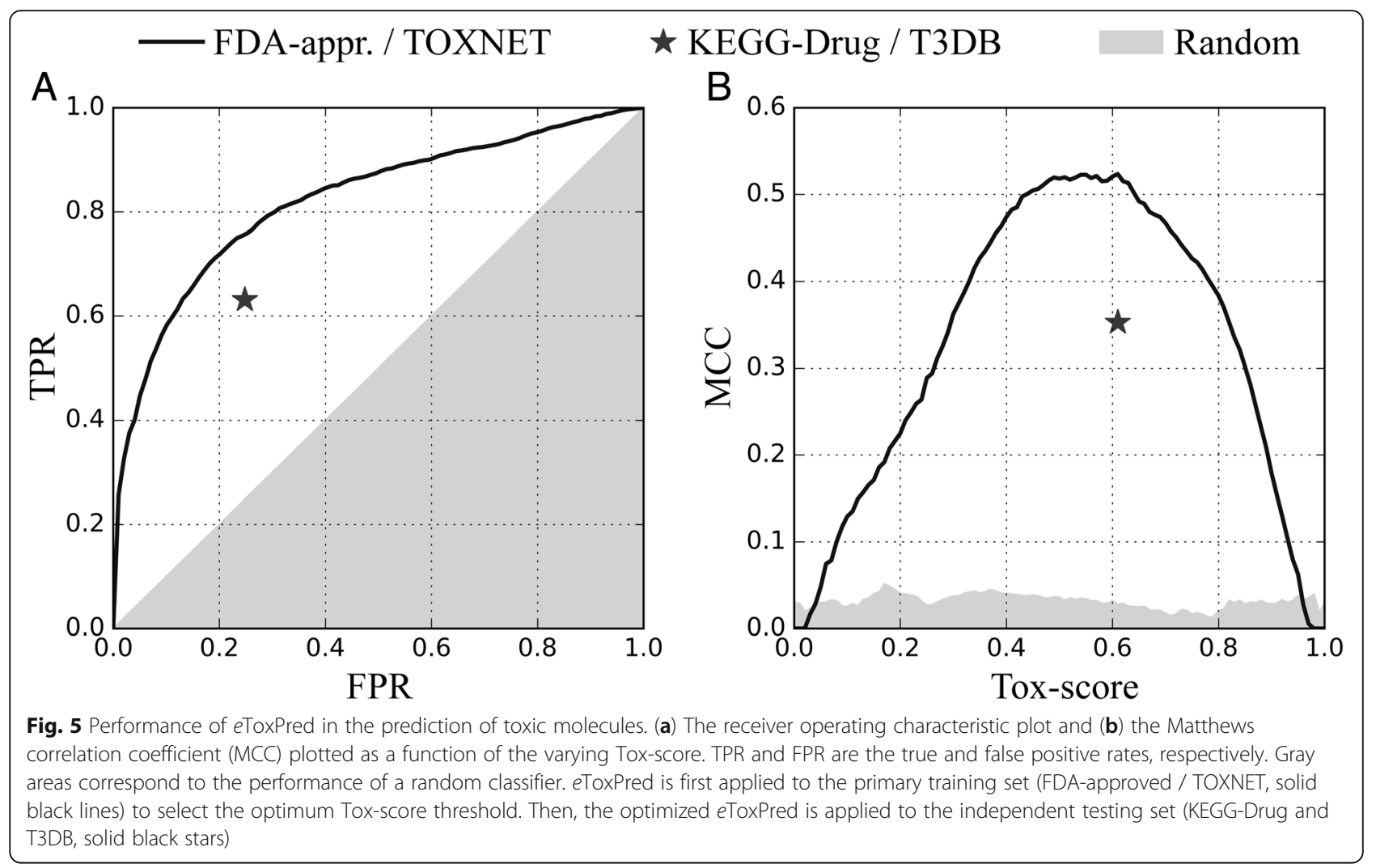

with the area under the curve (AUC) of 0.82 . According to Fig. $5 \mathrm{~B}$, a Tox-score of 0.58 the most effectively discriminates between toxic and non-toxic molecules, yielding an MCC (Eq. 4) of 0.52. Employing this threshold gives a high TPR of 0.71 at a low FPR of 0.19 .

Next, we apply $e$ ToxPred with the optimized Tox-score threshold to an independent dataset consisting of KEGG-Drug molecules, regarded as non-toxic, and toxic substances obtained from T3DB. Despite the fact that many of these compounds are unseen to the ET classifier, $e$ ToxPred quite efficiently recognizes toxic molecules. The MCC for the KEGG-Drug and T3DB datasets is 0.35 , corresponding to the TPR and FPR of 0.63 and 0.25 , respectively. Table 2 shows that using the ET classifier yields the best performance on this independent dataset compared to other machine learning techniques. Even though RF is slightly more accurate than ET against FDA-approved and TOXNET, the performance of ET is noticeably higher for KEGG-Drug and T3DB. In addition, we tested two other classifiers, the Linear Discriminant Analysis (LDA) [67] and Multilayer Perceptron (MLP) [68], however, their performance is generally not as high as those of RF and ET. Furthermore, the results obtained for the TCM dataset show that ET has the lowest tendency to over-predict the toxicity compared to other classifiers (the last row in Table 2).
Switching to an independent dataset causes the performance of machine learning classifiers to deteriorate on account of a fair amount of ambiguity in the training and testing sets. To better understand the datasets, we present a Venn diagram in Fig. 6. For instance, FDA-approved and TOXNET share as many as 559 molecules, whereas the intersection of KEGG-Drug and T3DB consists of 319 compounds. Further, 36 molecules classified as non-toxic in the FDA-approved / TOXNET dataset are labelled toxic in the KEGG-Drug / T3DB dataset (162 compounds are classified the other way around). As a result, the accuracy of both LDA and MLP drops from 0.74 to 0.65 , however, the accuracy of ET only slightly decreases from 0.76 to 0.72 , demonstrating the robustness of this classifier. Indeed, ET was previously shown to be resilient to high-noise conditions [43], therefore, we decided to employ this machine learning technique as a default classifier in $e$ ToxPred.

We also apply $e$ Toxpred to evaluate the compound toxicity across several datasets used to predict the synthetic accessibility. Not surprisingly, Fig. 4B shows that FDA-approved drugs have the lowest median Tox-score of 0.34. The toxicity of active compounds from the DUD-E dataset is a bit higher with a median Tox-score of 0.46. Molecules in both natural products datasets as well as traditional Chinese medicines are assigned even higher toxicity values; the median Tox-score is 0.56 , 
Table 2 Performance of various machine learning classifiers to predict toxicity. The following classifiers are tested

\begin{tabular}{llllll}
\hline Dataset & Metric & Toxicity classifiers & & \\
\cline { 3 - 5 } & & LDA & MLP & RF \\
\hline FDA-appr. / & ACC & 0.745 & 0.744 & $\mathbf{0 . 7 6 0}$ & 0.756 \\
TOXNET & TPR / FPR & $0.723 / 0.232$ & $0.679 / 0.180$ & $0.733 / 0.218$ & $0.719 / 0.186$ \\
& MCC & 0.495 & 0.525 & $\mathbf{0 . 5 2 8}$ & 0.523 \\
KEGG-Drug / & ACC & 0.647 & 0.645 & 0.674 & $\mathbf{0 . 7 2 1}$ \\
T3DB & TPR / FPR & $0.671 / 0.362$ & $0.675 / 0.365$ & $0.688 / 0.331$ & $0.631 / 0.248$ \\
& MCC & 0.272 & 0.273 & 0.316 & $\mathbf{0 . 3 5 3}$ \\
TCM & Tox-score & $0.504 \pm 0.013$ & $0.537 \pm 0.242$ & $0.574 \pm 0.143$ & $0.552 \pm 0.122$ \\
& \% toxic & 63.9 & 61.8 & 68.5 & 59.7 \\
\hline
\end{tabular}

Linear Discriminant Analysis (LDA), Multi-Layer Perceptron (MLP), Random Forest (RF), and Extra Trees (ET). Individual models are first trained and 5-fold crossvalidated against FDA-approved and TOXNET datasets and then applied to KEGG-Drug and T3DB as an additional validation against independent datasets. The performance of toxicity classifiers on FDA-approved / TOXNET and KEGG-Drug / T3DB datasets is assessed with the accuracy (ACC, Eq. 1), true (TPR, Eq. 2) and false (FPR, Eq. 3) positive rates, and the Matthews correlation coefficient (MCC, Eq. 4). The best performance across all models in terms of the highest ACC and MCC values are highlighted in bold. Finally, the trained models are applied to estimate the toxicity of traditional Chinese medicines in the TCM dataset and the average \pm standard deviation Tox-score values as well as the percentage of predicted toxic molecules are reported

0.54, and 0.54 for NuBBE, UNPD, and TCM, respectively. These results are in line with other studies examining the composition and toxicology of TCM, for instance, toxic constituents from various TCM sources include alkaloids, glycosides, peptides, amino acids, phenols, organic acids, terpenes, and lactones [69].

Finally, the prediction of specific toxicities is assessed against four independent datasets. Figure 7 and Table 3 show that the performance of $e$ ToxPred is the highest against the $\mathrm{AO}$ and $\mathrm{CD}$ datasets with AUC values of 0.80 . The performance against the remaining datasets, CP (AUC of 0.72) and ED (AUC of 0.75), is only slightly lower. These results are in line with benchmarking data reported for other classifiers; for instance, $e$ ToxPred compares favorably with different methods particularly against the $\mathrm{AO}$ and $\mathrm{ED}$ datasets [30, 70]. Importantly, the ET-based classifier employing molecular fingerprints

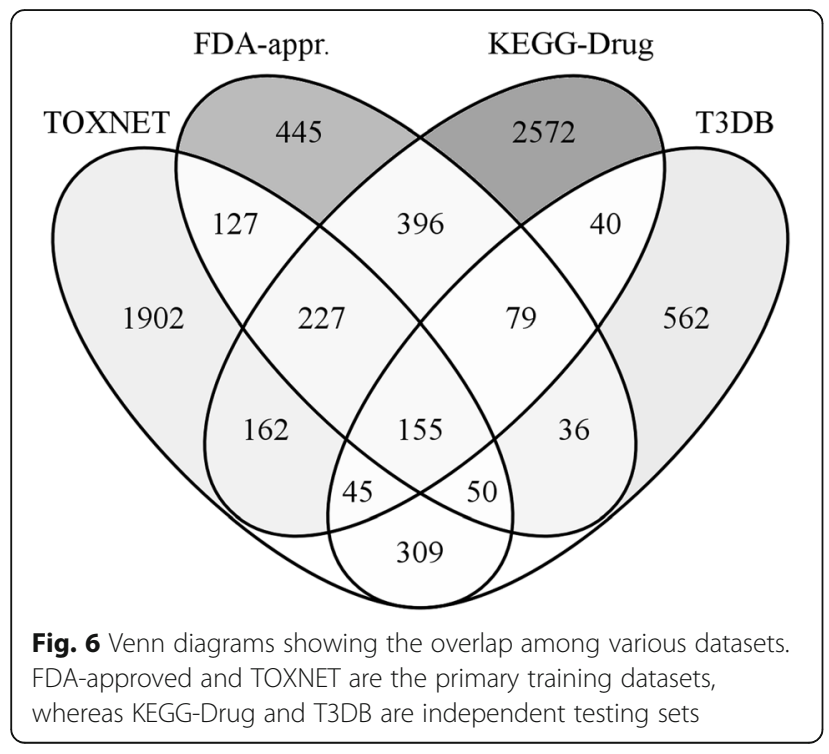

turns out to be highly effective predicting not only the general toxicity, but also specific toxicities as demonstrated for the carcinogenicity potency, cardiotoxicity, endocrine disruption, and acute oral toxicity.

\section{Composition of non-toxic compounds}

Since $e$ ToxPred quite effectively estimates the toxicity of small organic compounds from their molecular fingerprints, there should be some discernible structural attributes of toxic and non-toxic substances. On that account, we decomposed FDA-approved and TOXNET molecules into chemical fragments with eMolFrag [9] in order to compare their frequencies in both datasets.

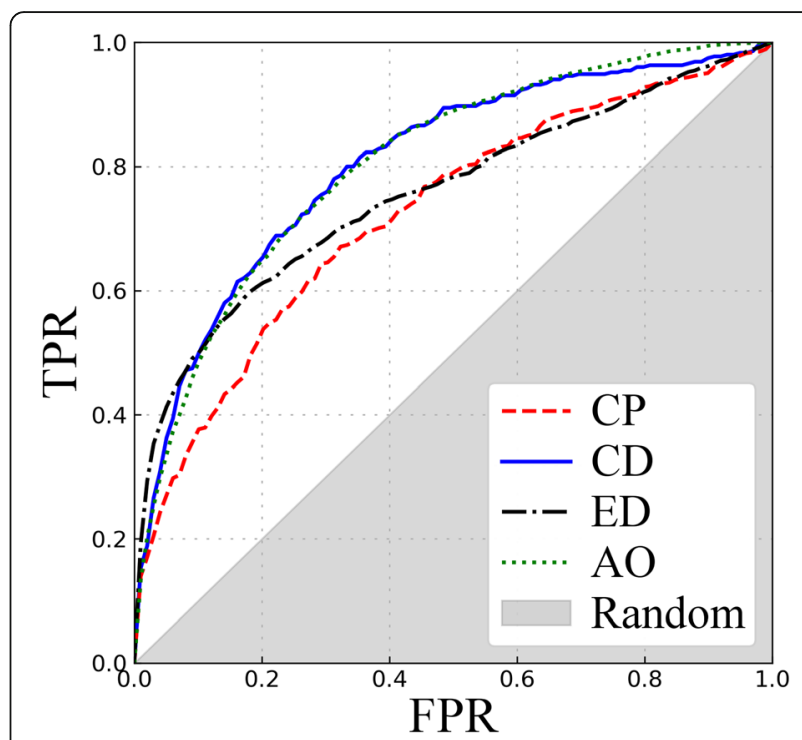

Fig. 7 Performance of eToxPred in the prediction of specific toxicities. The receiver operating characteristic plots are shown for Carcinogenicity Potency (CP), cardiotoxicity (CD), endocrine disruption (ED), and acute oral toxicity (AO) 
Table 3 Performance of the Extra Trees classifier to predict specific toxicities

\begin{tabular}{lll}
\hline Dataset & AUC & ACC \\
\hline CP & 0.721 & 0.722 \\
CD & 0.799 & 0.798 \\
ED & 0.750 & 0.744 \\
AO & 0.800 & 0.854 \\
\hline
\end{tabular}

The following datasets are used: carcinogenicity potency (CP), cardiotoxicity $(C D)$, endocrine disruption (ED), and acute oral toxicity (AO). The performance is assessed with the area under the curve (AUC) and the accuracy (ACC, Eq. 1)

Figure 8 shows a scatter plot of 698 distinct fragments extracted by $e$ MolFrag. As expected, the most common moiety is a benzene ring, whose frequency is 0.27 in the FDA-approved and 0.17 in TOXNET fragment sets. In general, fragment frequencies are highly correlated with a PCC of 0.98 , however, certain fragments are more often found in either dataset. To further investigate these cases, we selected three examples of fragments more commonly found in FDA-approved molecules, represented by green dots below the regression line in Fig. 8, and three counter examples of those fragments that are more frequent in the TOXNET dataset, shown as red dots above the regression line in Fig. 8. In addition, the selected parent molecules for these fragments are presented in Fig. 9 (FDA-approved) and Fig. 10 (TOXNET).

Examples shown in Fig. 9 include piperidine (Fig. 9A), piperazine (Fig. 9B), and fluorophenyl (Fig. 9C) moieties, whose frequencies in FDA-approved/TOXNET datasets are $0.069 / 0.026,0.032 / 0.010$, and $0.024 / 0.007$, respectively. Nitrogen-bearing heterocycles, piperidine and piperazine, are of central importance to medicinal chemistry [71]. Piperidine offers a number of important functionalities that have been exploited to develop central nervous system modulators, anticoagulants, antihistamines, anticancer agents and analgesics [72]. This scaffold is the basis for over 70 drugs, including those shown in Fig. 9A, trihexyphenidyl (DrugBank-ID: DB00376), a muscarinic antagonist to treat Parkinson's disease [73], donepezil (DrugBank-ID: DB00843), a reversible acetyl cholinesterase inhibitor to treat Alzheimer's disease [74], an opioid analgesic drug remifentanil (DrugBank-ID: DB00899) [75], and dipyridamole (DrugBank-ID: DB00975), a phosphodiesterase inhibitor preventing the blood clot formation [76].

Similarly, many well established and commercially available drugs contain a piperazine ring as part of their molecular structures [77]. A wide array of pharmacological

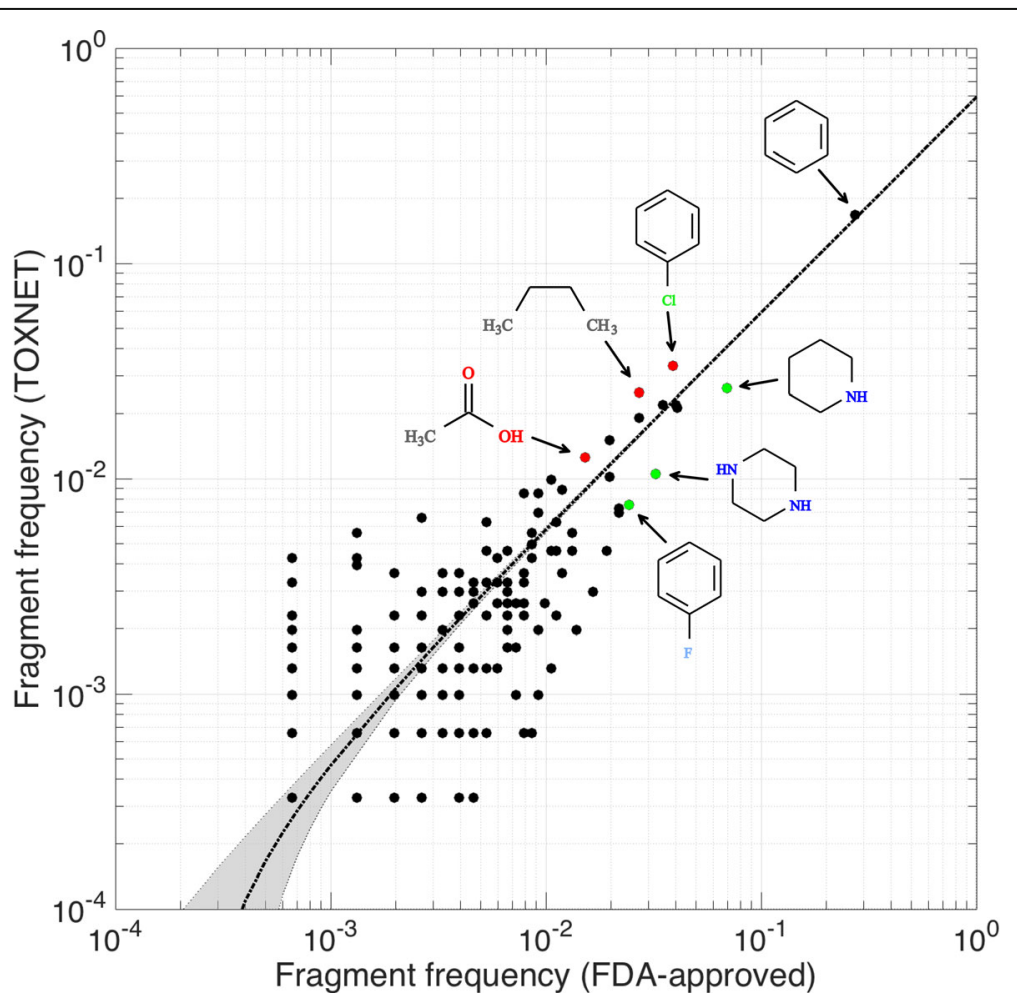

Fig. 8 Composition of non-toxic and toxic compounds. The scatter plot compares the frequencies of chemical fragments extracted with eMolFrag from FDA-approved (non-toxic) and TOXNET (toxic) molecules. The regression line is dotted black and the gray area delineates the corresponding confidence intervals. Three selected examples of fragments more commonly found in FDA-approved molecules (piperidine, piperazine, and fluorophenyl) are colored in green, whereas three counter examples of fragments more frequent in the TOXNET dataset (chlorophenyl, $n$-butyl, and acetic acid) are colored in red 
A<smiles>C1CCNCC1</smiles><smiles>c1ccc(CC[C](c2ccccc2)c2ccccc2)cc1</smiles>

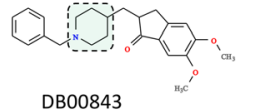

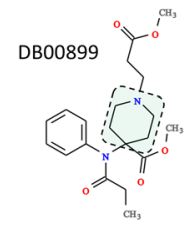

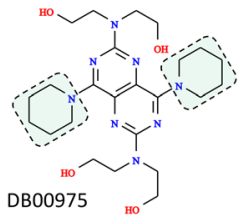

B<smiles>C1CNCCN1</smiles>

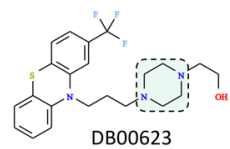

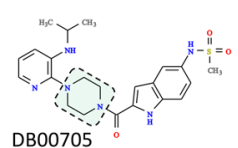<smiles>Cc1cccc(Cc2ccc(C(c3ccccc3)c3ccc(O)cc3)cc2)c1</smiles>

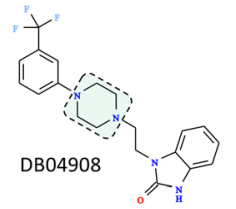<smiles>Fc1ccccc1</smiles>

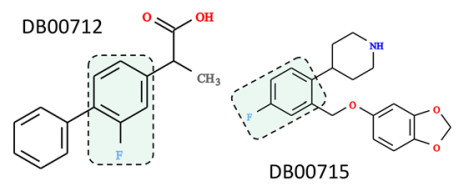

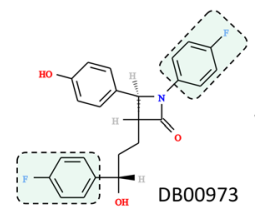

Fig. 9 Composition of selected non-toxic compounds. Three examples of fragments more commonly found in FDA-approved molecules than in the TOXNET dataset: (a) piperidine, (b) piperazine, and (c) fluorophenyl. Four sample molecules containing a particular moiety (highlighted by green boxes) are selected from DrugBank and labeled by the DrugBank-ID

activities exhibited by piperazine derivatives make them attractive leads to develop new antidepressant, anticancer, anthelmintic, antibacterial, antifungal, antimalarial, and anticonvulsant therapeutics [78]. Selected examples of piperazine-based drugs presented in Fig. 9B, are antipsychotic fluphenazine (DrugBank-ID: DB00623), antiretroviral delavirdine (DrugBank-ID: DB00705), antihistamine meclizine (DrugBank-ID: DB00737), and flibanserin (DrugBank-ID: DB04908) to treat hypoactive sexual desire disorder among pre-menopausal women [79]. All of these compounds contain substituents at both N1- and N4-positions, which concurs with the analysis of piperazine substitution patterns across FDA-approved pharmaceuticals revealing that $83 \%$ of piperazine-containing drugs are substituted at both nitrogens, whereas only a handful have a substituent at any other position [77].

Incorporating fluorine into drug leads is an established practice in drug design and optimization. In fact, so-called fluorine scan is often employed in the development of drug candidates to systematically exploit the benefits of

A<smiles>CC(=O)c1cc2c3c(c1)C[C](C2)C3</smiles>

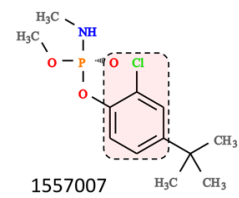

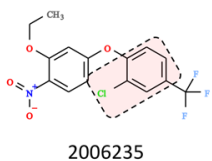

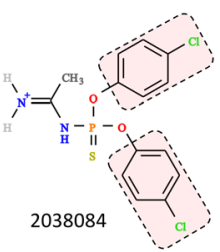

B<smiles>CCCC</smiles>

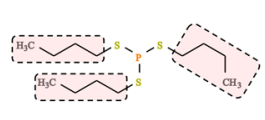

1641617<smiles>[B]C(C)(O)C(=O)OCCCCCCCCC</smiles>

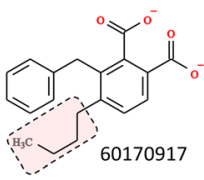<smiles>CC(=O)O</smiles>

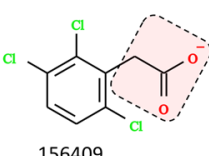<smiles>CCCCCCOC(=O)OC1CCC(Cl)CC1</smiles>

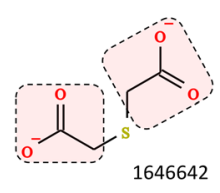

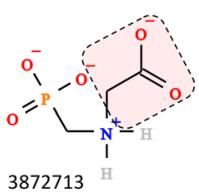

Fig. 10 Composition of selected toxic compounds. Three examples of fragments more commonly found in the TOXNET dataset than in FDAapproved molecules: (a) chlorophenyl, (b) n-butyl, and (c) acetic acid. Four sample molecules containing a particular moiety (highlighted by red boxes) are selected from ZINC and labeled by the ZINC-ID 
fluorine substitution [80]. As a result, an estimated one-third of the top-performing drugs currently on the market contain fluorine atoms in their structure [81]. The presence of fluorine atoms in pharmaceuticals increases their bioavailability by modulating $\mathrm{p} K_{\mathrm{a}}$ and lipophilicity, as well as by improving their absorption and partitioning into membranes [82]. Further, fluorination helps stabilize the binding of a drug to a protein pocket by creating additional favorable interactions, as it was suggested for the fluorophenyl ring of paroxetine (DrugBank-ID: DB00715) [83], a selective serotonin reuptake inhibitor shown in Fig. 9C. A low metabolic stability due to cytochrome P450-mediated oxidation can be mitigated by blocking metabolically unstable hydrogen positions with fluorine atoms [84], as exemplified by drug structures shown in Fig. 9C. Indeed, a targeted fluorination of a nonsteroidal anti-inflammatory drug flurbiprofen (DrugBank-ID: DB00712) helped prolong its metabolic half-life [85]. Another example is cholesterol inhibitor ezetimibe (DrugBank-ID: DB00973), in which two metabolically labile sites are effectively blocked by fluorine substituents [86]. Finally, replacing the chlorine atom with a fluorine improves safety profile and pharmacokinetic properties of prasugrel (DrugBank-ID: DB06209) compared to other thienopyridine antiplatelet drugs, ticlopidine and clopidogrel [87].

\section{Composition of toxic compounds}

Next, we selected three counter examples (red dots in Fig. 8) of fragments frequently found in toxic substances, chlorophenyl, $n$-butyl, and acetic acid, whose representative parent molecules are presented in Fig. 10. For instance, the chlorophenyl moiety (Fig. 10A) is the constituent of $p$-chloroacetophenone (ZINC-ID: 896324) used as a tear gas for riot control, crufomate (ZINC-ID: 1557007), an insecticide potentially toxic to humans, the herbicide oxyfluorfen (ZINC-ID: 2006235), and phosacetim (ZINC-ID: 2038084), a toxic acetylcholinesterase inhibitor used as a rodenticide. Further, $n$-butyl groups (Fig. 10B) are present in a number of toxic substances, including merphos (ZINC-ID: 1641617), a pesticide producing a delayed neurotoxicity in animals, $n$-butyl lactate (ZINC-ID: 1693581), an industrial chemical and food additive, diethylene glycol monobutyl ether acetate (ZINC-ID: 34958085) used as solvents for cleaning fluids, paints, coatings and inks, and $n$-butyl benzyl phthalate (ZINC-ID: 60170917), a plasticizer for vinyl foams classified as toxic in Europe and excluded from the manufacturing of toys and child care products in Canada. The last example is the acetic acid moiety (Fig. 10C) found in many herbicides, e.g. chlorfenac (ZINC-ID: 156409), 4-chlorophenoxyacetic acid (ZINC-ID: 347851), and glyphosate (ZINC-ID: 3872713) as well as in thiodiacetic acid (ZINC-ID: 1646642), a chemical used by the material industry to synthesize sulfur-based electroconductive polymers.

\section{Conclusions}

In this study, we developed a new program to predict the synthetic accessibility and toxicity of small organic compounds directly from their molecular fingerprints. The estimated toxicity is reported as the Tox-score, a new machine learning-based scoring metric implemented in eToxPred, whereas the synthetic accessibility is evaluated with the SAscore, an already established measure in this field. We previously developed tools, such as $e$ MolFrag and $e$ Synth, to build large, yet target-specific compound libraries for virtual screening. $e$ ToxPred can be employed as a post-generation filtering step to eliminate molecules that are either difficult to synthesize or resemble toxic substances included in TOXNET and T3DB rather than FDA-approved drugs and compounds listed by the KEGG-Drug dataset. Additionally, it effectively predicts specific toxicities, such as the carcinogenicity potency, cardiotoxicity, endocrine disruption, and acute oral toxicity. In principle, this procedure could save considerable resources by concentrating the subsequent virtual screening and molecular modeling simulations on those compounds having a better potential to become leads.

\section{Availability and requirements \\ Project name: $e$ ToxPred. \\ Project home page: https://github.com/pulimeng/ etoxpred \\ Operating system(s): Platform independent. \\ Programming language: Python 2.7+ or Python 3.5+. \\ Other requirements: Theano, numpy 1.8.2 or higher, scipy 0.13 .3 or higher, scikit-learn 0.18.1, OpenBabel 2.3.1, CUDA 8.0 or higher (optional).}

\section{License: GNU GPL.}

Any restrictions to use by non-academics: license needed.

\section{Abbreviations \\ ACC: accuracy; ADMET: absorption, distribution, metabolism, excretion, and toxicity; CADD: computer-aided drug discovery; DBN: deep belief network; DNN: deep neural network; DUD-E: Database of Useful Decoys, Extended; ET: extra trees; FDA: Food and Drug Administration; FPR: false positive rate; GPU: graphics processing units; hERG: human Ether-à-go-go-Related Gene; KEGG: Kyoto Encyclopedia of Genes and Genomes; LBDD: ligand-based drug design; LD: lethal dose; LDA: Linear Discriminant Analysis; MCC: Matthews correlation coefficient; MLP: Multilayer Perceptron; MSE: mean squared error; NuBBE: Nuclei of Bioassays, Ecophysiology and Biosynthesis of Natural Products; PCC: Pearson correlation coefficient; QSAR: quantitative structure- activity relationship; QSPR: quantitative-structure property relationships; RBM: restricted Boltzmann machine; RF: random forest; ROC: Receiver Operating Characteristic; SBDD: structure-based drug design; SVM: support vector machine; T3DB: Toxin and Toxin Target Database; TC: Tanimoto coefficient; TCM: Traditional Chinese Medicine; TOPKAT: TOxicity Prediction by Komputer Assisted Technology; TPR: true positive rate; UNPD: Universal Natural Products Database; VS: virtual screening}

\section{Acknowledgements}

The authors are grateful to Louisiana State University for providing computing resources. 


\section{Availability of data and material}

Datasets are freely available to the community through the Open Science Framework at https://osf.io/m4ah5/.

\section{Funding}

Research reported in this publication was supported by the National Institute of General Medical Sciences of the National Institutes of Health under Award Number R35GM119524.

\section{Authors' contributions}

LP implemented eToxPred, performed calculations and validation. MN and MB prepared datasets. TL decomposed molecules into chemical fragments. $L P, M N$, TL, and $M B$ analyzed results. HCW and SM contributed algorithms. LP drafted the manuscript. MB coordinated the project and prepared the final version of the manuscript

\section{Ethics approval and consent to participate}

Not applicable.

\section{Consent for publication}

Not applicable.

\section{Competing interests}

Michal Brylinski serves as Associate Editor for BMC Pharmacology and Toxicology.

\section{Publisher's Note}

Springer Nature remains neutral with regard to jurisdictional claims in published maps and institutional affiliations.

\section{Author details}

'Division of Electrical \& Computer Engineering, Louisiana State University, Baton Rouge, LA 70803, USA. ²Department of Biological Sciences, Louisiana State University, Baton Rouge, LA 70803, USA. ${ }^{3}$ Department of Mechanical Engineering, Louisiana State University, Baton Rouge, LA 70803, USA. ${ }^{4}$ Department of Computer Science, Louisiana State University, Baton Rouge, LA 70803, USA. ${ }^{5}$ Center for Computation \& Technology, Louisiana State University, Baton Rouge, LA 70803, USA.

\section{Received: 14 August 2018 Accepted: 26 December 2018}

\section{Published online: 08 January 2019}

\section{References}

1. DiMasi JA, Grabowski HG, Hansen RW. Innovation in the pharmaceutical industry: new estimates of R\&D costs. J Health Econ. 2016:47:20-33.

2. Paul SM, Mytelka DS, Dunwiddie CT, Persinger CC, Munos BH, Lindborg SR, Schacht AL. How to improve R\&D productivity: the pharmaceutical industry's grand challenge. Nat Rev Drug Discov. 2010;9:203-14.

3. Hung CL, Chen CC. Computational approaches for drug discovery. Drug Dev Res. 2014;75:412-8.

4. Sliwoski G, Kothiwale S, Meiler J, Lowe EW Jr. Computational methods in drug discovery. Pharmacol Rev. 2014;66:334-95.

5. Acharya C, Coop A, Polli JE, Mackerell AD Jr. Recent advances in ligandbased drug design: relevance and utility of the conformationally sampled pharmacophore approach. Curr Comput Aided Drug Des. 2011;7:10-22.

6. Yang SY. Pharmacophore modeling and applications in drug discovery: challenges and recent advances. Drug Discov Today. 2010;15:444-50.

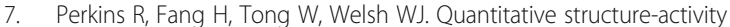
relationship methods: perspectives on drug discovery and toxicology. Environ Toxicol Chem. 2003;22:1666-79.

8. Chevillard F, Kolb P. SCUBIDOO: a large yet screenable and easily searchable database of computationally created chemical compounds optimized toward high likelihood of synthetic tractability. J Chem Inf Model. 2015;55: 1824-35.

9. Liu T, Naderi M, Alvin C, Mukhopadhyay S, Brylinski M. Break down in order to build up: decomposing small molecules for fragment-based drug design with eMolFrag. J Chem Inf Model. 2017:57:627-31.

10. Naderi M, Alvin C, Ding Y, Mukhopadhyay S, Brylinski M. A graph-based approach to construct target-focused libraries for virtual screening. J Cheminform. 2016;8:14
11. Cushman DW, Ondetti MA. History of the design of captopril and related inhibitors of angiotensin converting enzyme. Hypertension. 1991;17:589-92.

12. Braga RC, Andrade CH. Assessing the performance of $3 \mathrm{D}$ pharmacophore models in virtual screening: how good are they? Curr Top Med Chem. 2013 13:1127-38.

13. Kim KH, Kim ND, Seong BL. Pharmacophore-based virtual screening: a review of recent applications. Expert Opin Drug Discov. 2010;5:205-22.

14. Anderson AC. The process of structure-based drug design. Chem Biol. 2003; 10:787-97.

15. Morris GM, Lim-Wilby M. Molecular docking. Methods Mol Biol. 2008;443: 365-82.

16. De Vivo M, Masetti M, Bottegoni G, Cavalli A. Role of molecular dynamics and related methods in drug discovery. J Med Chem. 2016;59:4035-61.

17. Cerqueira NM, Gesto D, Oliveira EF, Santos-Martins D, Bras NF, Sousa SF, Fernandes PA, Ramos MJ. Receptor-based virtual screening protocol for drug discovery. Arch Biochem Biophys. 2015;582:56-67.

18. Schneider G, Fechner U. Computer-based de novo design of drug-like molecules. Nat Rev Drug Discov. 2005;4:649-63.

19. Trott O, Olson AJ. AutoDock Vina: improving the speed and accuracy of docking with a new scoring function, efficient optimization, and multithreading. J Comput Chem. 2010;31:455-61.

20. Ruiz-Carmona S, Alvarez-Garcia D, Foloppe N, Garmendia-Doval AB, Juhos S, Schmidtke P, Barril X, Hubbard RE, Morley SD. rDock: a fast, versatile and open source program for docking ligands to proteins and nucleic acids. PLoS Comput Biol. 2014;10:e1003571.

21. Friesner RA, Banks JL, Murphy RB, Halgren TA, Klicic JJ, Mainz DT, Repasky MP, Knoll EH, Shelley M, Perry JK, et al. Glide: a new approach for rapid, accurate docking and scoring. 1. Method and assessment of docking accuracy. J Med Chem. 2004;47:1739-49.

22. Schellhammer I, Rarey M. FlexX-scan: fast, structure-based virtual screening. Proteins. 2004;57:504-17

23. Singh J, Chuaqui CE, Boriack-Sjodin PA, Lee WC, Pontz T, Corbley MJ, Cheung HK, Arduini RM, Mead JN, Newman MN, et al. Successful shapebased virtual screening: the discovery of a potent inhibitor of the type I TGFbeta receptor kinase (TbetaRI). Bioorg Med Chem Lett. 2003;13:4355-9.

24. Sawyer JS, Anderson BD, Beight DW, Campbell RM, Jones ML, Herron DK, Lampe JW, McCowan JR, McMillen WT, Mort N, et al. Synthesis and activity of new aryl- and heteroaryl-substituted pyrazole inhibitors of the transforming growth factor-beta type I receptor kinase domain. J Med Chem. 2003:46:3953-6.

25. Sanguinetti MC, Tristani-Firouzi M. hERG potassium channels and cardiac arrhythmia. Nature. 2006:440:463-9.

26. Braga RC, Alves VM, Silva MF, Muratov E, Fourches D, Liao LM, Tropsha A, Andrade $\mathrm{CH}$. Pred-hERG: a novel web-accessible computational tool for predicting cardiac toxicity. Mol Inform. 2015;34:698-701.

27. Mortelmans K, Zeiger E. The Ames salmonella/microsome mutagenicity assay. Mutat Res. 2000:455:29-60.

28. Drwal MN, Banerjee P, Dunkel M, Wettig MR, Preissner R. ProTox: a web server for the in silico prediction of rodent oral toxicity. Nucleic Acids Res. 2014;42:W53-8.

29. Raies $A B$, Bajic VB. In silico toxicology: computational methods for the prediction of chemical toxicity. Wiley Interdiscip Rev Comput Mol Sci. 2016; 6:147-72

30. Mayr A, Klambauer G, Unterthiner T, Hochreiter S. DeepTox: toxicity prediction using deep learning. Front Environ Sci. 2016;3.

31. Schmidhuber J. Deep learning in neural networks: an overview. Neural Netw. 2015:61:85-117.

32. Rosenbaum L, Hinselmann G, Jahn A, Zell A. Interpreting linear support vector machine models with heat map molecule coloring. J Cheminform 2011;3:11.

33. Breiman L. Random forests. Mach Learn. 2001;45:61-3.

34. Friedman J, Hastie T, Tibshirani R. Regularization paths for generalized linear models via coordinate descent. J Stat Softw. 2010;33:1-22.

35. Chaudhari R, Tan Z, Huang B, Zhang S. Computational polypharmacology: a new paradigm for drug discovery. Expert Opin Drug Discov. 2017;12:279-91.

36. Ackley $\mathrm{DH}$, Hinton GE, Sejnowski TJ. A learning algorithm for boltzmann machines. Cognitive Sci. 1985;9:147-69.

37. Smolensky P. Information processing in dynamical systems: foundations of harmony theory. In: Rumelhart DE, McClelland JL, editors. Parallel distributed processing: explorations in the microstructure of cognition, vol. 1. Cambridge, MA: MIT Press; 1986. p. 194-281. 
38. Geman S, Geman D. Stochastic relaxation, Gibbs distributions, and the bayesian restoration of images. IEEE Trans Pattern Anal Mach Intell. 1984;6:721-41.

39. Hinton GE. Deep belief networks. Scholarpedia. 2009;4:5947.

40. Hinton GE. Training products of experts by minimizing contrastive divergence. Neural Comput. 2002;14:1771-800.

41. Bengio Y. Learning deep architectures for Al. Foundations and Trends in Machine Learning. 2009;2:1-127.

42. Theano_Development_Team: Theano: A Python framework for fast computation of mathematical expressions. arXiv e-prints 2016:abs/ 1605.02688

43. Geurts $P$, Ernst $D$, Wehenkel L. Extremely randomized trees. Mach Learn. 2006;63:3-42.

44. Ho TK. Random decision forests. Third Int'I Conf Document Analysis and Recognition. 1995:278-82.

45. Breiman L, Friedman JH, Olshen RA, Stone CJ. Classification and regression trees. Belmont, CA: Wadsworth; 1984.

46. Valli M, dos Santos RN, Figueira LD, Nakajima CH, Castro-Gamboa I, Andricopulo AD, Bolzani VS. Development of a natural products database from the biodiversity of Brazil. J Nat Prod. 2013;76:439-44.

47. Gu J, Gui Y, Chen L, Yuan G, Lu HZ. Xu X: use of natural products as chemical library for drug discovery and network pharmacology. PLoS One. 2013:8:e62839.

48. Tanimoto TT. An elementary mathematical theory of classification and prediction. In: Book An elementary mathematical theory of classification and prediction. (editor ed.^eds.). City; 1958.

49. Voigt JH, Bienfait B, Wang S, Nicklaus MC. Comparison of the $\mathrm{NCl}$ open database with seven large chemical structural databases. J Chem Inf Comput Sci. 2001;41:702-12.

50. Mysinger MM, Carchia M, Irwin JJ, Shoichet BK. Directory of useful decoys, enhanced (DUD-E): better ligands and decoys for better benchmarking. J Med Chem. 2012;55:6582-94.

51. Wishart DS, Knox C, Guo AC, Shrivastava S, Hassanali M, Stothard P, Chang Z, Woolsey J. DrugBank: a comprehensive resource for in silico drug discovery and exploration. Nucleic Acids Res. 2006;34:D668-72.

52. Kanehisa M, Goto S, Furumichi M, Tanabe M, Hirakawa M. KEGG for representation and analysis of molecular networks involving diseases and drugs. Nucleic Acids Res. 2010;38:D355-60.

53. Wexler P. TOXNET: the National Library of Medicine's toxicology database. Am Fam Physician. 1995;52:1677-8.

54. Lim E, Pon A, Djoumbou Y, Knox C, Shrivastava S, Guo AC, Neveu V, Wishart DS. T3DB: a comprehensively annotated database of common toxins and their targets. Nucleic Acids Res. 2010;38:D781-6.

55. Chen CY. TCM database@Taiwan: the world's largest traditional Chinese medicine database for drug screening in silico. PLoS One. 2011;6:e15939.

56. Gold LS, Slone TH, Ames BN. Overview of analyses of the carcinogenic potency database. In: Gold LS, Zeiger E, editors. Handbook of carcinogenic potency and genotoxicity databases. Boca Raton, FL: CRC Press; 1997. p. 661-85.

57. Du L, Li M, You Q. The interactions between hERG potassium channel and blockers. Curr Top Med Chem. 2009:9:330-8.

58. Wang S, Li Y, Wang J, Chen L, Zhang L, Yu H, Hou T. ADMET evaluation in drug discovery. 12. Development of binary classification models for prediction of hERG potassium channel blockage. Mol Pharm. 2012;9:996-1010.

59. Lee HR, Jeung EB, Cho MH, Kim TH, Leung PC, Choi KC. Molecular mechanism(s) of endocrine-disrupting chemicals and their potent oestrogenicity in diverse cells and tissues that express oestrogen receptors. J Cell Mol Med. 2013:17:1-11.

60. Schmidt U, Struck S, Gruening B, Hossbach J, Jaeger IS, Parol R, Lindequist $U$, Teuscher E, Preissner R. SuperToxic: a comprehensive database of toxic compounds. Nucleic Acids Res. 2009;37:D295-9.

61. O'Boyle NM, Banck M, James CA, Morley C, Vandermeersch T, Hutchison GR Open babel: an open chemical toolbox. J Cheminform. 2011;3:33.

62. Ertl P, Schuffenhauer A. Estimation of synthetic accessibility score of druglike molecules based on molecular complexity and fragment contributions. J Cheminform. 2009;1:8.

63. Geisser S. Predictive inference. New York, NY: Chapman and Hall; 1993

64. James $G$, Witten D, Hastie T, Tibshirani R. An introduction to statistical learning. New York: Springer-Verlag New York; 2013.

65. Matthews BW. Comparison of the predicted and observed secondary structure of T4 phage lysozyme. Biochim Biophys Acta. 1975;405:442-51.
66. Pearson K. VII. Note on regression and inheritance in the case of two parents. Proc Royal Soc London. 1895:58:240-2.

67. McLachlan G. Discriminant analysis and statistical. Pattern Recogn. 2004.

68. Rosenblatt F. Principles of neurodynamics; perceptrons and the theory of brain mechanisms; 1962

69. Lv W, Piao JH, Jiang JG. Typical toxic components in traditional Chinese medicine. Expert Opin Drug Saf. 2012;11:985-1002.

70. Li X, Chen L, Cheng F, Wu Z, Bian H, Xu C, Li W, Liu G, Shen X, Tang Y. In silico prediction of chemical acute oral toxicity using multi-classification methods. J Chem Inf Model. 2014;54:1061-9.

71. Taylor RD, MacCoss M, Lawson AD. Rings in drugs. J Med Chem. 2014;57: 5845-59.

72. Vardanyan R: Chapter 10 - Classes of piperidine-based drugs. In Piperidinebased drug discovery. Elsevier; 2017: 299-332: Heterocyclic Drug Discovery].

73. Muenter MD, Dinapoli RP, Sharpless NS, Tyce GM. 3-O-methyldopa, L-dopa, and trihexyphenidyl in the treatment of Parkinson's disease. Mayo Clin Proc. 1973:48:173-83.

74. Tan CC, Yu JT, Wang HF, Tan MS, Meng XF, Wang C, Jiang T, Zhu XC, Tan L. Efficacy and safety of donepezil, galantamine, rivastigmine, and memantine for the treatment of Alzheimer's disease: a systematic review and metaanalysis. J Alzheimers Dis. 2014;41:615-31.

75. Patel SS, Spencer CM. Remifentanil. Drugs. 1996:52:417-27 discussion 428

76. Diener HC, Cunha L, Forbes C, Sivenius J, Smets P, Lowenthal A. European stroke prevention study. 2. Dipyridamole and acetylsalicylic acid in the secondary prevention of stroke. J Neurol Sci. 1996;143:1-13.

77. Vitaku E, Smith DT, Njardarson JT. Analysis of the structural diversity, substitution patterns, and frequency of nitrogen heterocycles among U.S. FDA approved pharmaceuticals. J Med Chem. 2014;57:10257-74.

78. Shaquiquzzaman M, Verma G, Marella A, Akhter M, Akhtar W, Khan MF, Tasneem S, Alam MM. Piperazine scaffold: a remarkable tool in generation of diverse pharmacological agents. Eur J Med Chem. 2015;102:487-529.

79. Borsini F, Evans K, Jason K, Rohde F, Alexander B, Pollentier S. Pharmacology of flibanserin. CNS Drug Rev. 2002:8:117-42.

80. Hyohdoh I, Furuichi N, Aoki T, Itezono Y, Shirai H, Ozawa S, Watanabe F, Matsushita M, Sakaitani M, Ho PS, et al. Fluorine scanning by nonselective fluorination: enhancing Raf/MEK inhibition while keeping physicochemical properties. ACS Med Chem Lett. 2013;4:1059-63.

81. Wang J, Sanchez-Rosello M, Acena JL, del Pozo C, Sorochinsky AE, Fustero S, Soloshonok VA, Liu H. Fluorine in pharmaceutical industry: fluorinecontaining drugs introduced to the market in the last decade (2001-2011). Chem Rev. 2014;114:2432-506.

82. Purser S, Moore PR, Swallow S, Gouverneur V. Fluorine in medicinal chemistry. Chem Soc Rev. 2008;37:320-30

83. Davis BA, Nagarajan A, Forrest LR, Singh SK. Mechanism of paroxetine (paxil) inhibition of the serotonin transporter. Sci Rep. 2016;6:23789.

84. Bohm HJ, Banner D, Bendels S, Kansy M, Kuhn B, Muller K, Obst-Sander U, Stahl M. Fluorine in medicinal chemistry. Chembiochem. 2004;5:637-43.

85. Shaughnessy MJ, Harsanyi A, Li J, Bright T, Murphy CD, Sandford G. Targeted fluorination of a nonsteroidal anti-inflammatory drug to prolong metabolic half-life. ChemMedChem. 2014;9:733-6.

86. Van Heek M, France CF, Compton DS, McLeod RL, Yumibe NP, Alton KB, Sybertz EJ, Davis HR Jr. In vivo metabolism-based discovery of a potent cholesterol absorption inhibitor, SCH58235, in the rat and rhesus monkey through the identification of the active metabolites of SCH48461. J Pharmacol Exp Ther. 1997:283:157-63.

87. Reinhart KM, White CM, Baker WL. Prasugrel: a critical comparison with clopidogrel. Pharmacotherapy. 2009;29:1441-51.

Ready to submit your research? Choose BMC and benefit from:

- fast, convenient online submission

- thorough peer review by experienced researchers in your field

- rapid publication on acceptance

- support for research data, including large and complex data types

- gold Open Access which fosters wider collaboration and increased citations

- maximum visibility for your research: over $100 \mathrm{M}$ website views per year

At $\mathrm{BMC}$, research is always in progress.

Learn more biomedcentral.com/submission 